\title{
Thermal parameter extraction for screening procedure of skin pathologies based on the cold provocation
}

\author{
by M. Strąkowska*, M. Strzelecki*, A. Kaszuba** and B. Więcek*
}

\begin{abstract}
* Institute of Electronics Lodz University Of Technology, Lodz, Poland, strakowska.m@gmail.com **Department of Dermatology, Pediatric Dermatology and Dermatological Oncology Medical University of Lodz, Lodz, Poland, andrzej.kaszuba@umed.lodz.pl
\end{abstract}

\begin{abstract}
This paper presents a novel screening method based on thermal image classification. The classification uses the thermal parameters of the multilayer structure of the skin tissue as the images' features. These physical thermal parameters are evaluated using the inverse thermal modelling. The thermal process in the tissue was induced by the weak cooling. Temperature rise after cooling was used to recover the unknown thermal parameters including perfusion.
\end{abstract}

\section{Thermal modeling of a perfused tissue}

Cold provocation and thermal modeling of a tissue are known in medicine for years [1-8]. Among many thermal models, the Pennes one was the first and still is in use [1-8]. It assumes the heat transfer is tissue depended according the diffusion equation, but with the extension of extra component responsible for the perfusion - equation (1). Tissues are not the homogenous structures and the perfusion has to be taken into account. In addition, the metabolic heat can be taken in account as it plays not negligible role in the heat balance in the tissue [1-4]. In many practical cases skin tissue can be modelled as multilayer objects [6-8]. Modeling the multilayer thermal structures with blood flow and perfusion is not an easy task. Nowadays, the numerical modelling is preferred, but it needs powerful computing and it is timeconsuming. As a consequence it is not a real time approach and it cannot be used in the daily screening.

This model is valid for all the layers of the tissue $i=1,2,3$.

$$
\rho_{i} \cdot c_{p i} \cdot \frac{d T}{d t}=\lambda_{i} \cdot \frac{d^{2} T}{d x^{2}}+w_{i} \cdot c_{b i} \cdot \rho_{b i}\left(T_{b i}-T\right)+q_{m i}
$$

where: $\rho_{i}$ - tissue density (i-th layer), $c_{p i}$ - specific heat, $\lambda_{i}-$ thermal conductivity, $w_{i}-$ perfusion coefficient, $\rho_{b i}$, $c_{b i}-$ density and specific heat of the blood and $q_{m i}-$ metabolic heat in $i$-th layer.

By introducing the thermal capacity $C_{t h i}=\rho_{i} C_{p i}, C_{t h b i}=\rho_{b i} C_{b i}$, and assuming $T_{b i}=0$, one can rewrite the last equation.

$$
\lambda_{i} \frac{d^{2} T}{d x^{2}}-C_{t h i} \cdot \frac{d T}{d t}-w_{i} \cdot C_{t h b i} \cdot T=-q_{m i}
$$

Equation (2) can be presented in Laplace domain (3), and then it can be solved analytically [6-8]. In order to get the temperature field in all layers of the structure, one has to define the boundary and interface conditions between the layers. Then the set of linear equations is formed and solved either by analytical and numerical means [6-8].

$$
\begin{aligned}
& \lambda_{i} \frac{d^{2} T}{d x^{2}}-T\left(C_{t h i} \cdot s+w \cdot C_{t h b i}\right)=-q_{m i} \\
& \frac{d^{2} T}{d x^{2}}-\frac{T}{L_{i}^{2}}=-\frac{q_{m i}}{\lambda_{i}} \\
& L_{i}(s)=\frac{1}{\sqrt{\left(C_{t h i} \cdot s+w \cdot C_{t h b i}\right) / \lambda_{i}}}
\end{aligned}
$$

The problem of thermal modeling becomes more difficult if we consider inverse thermal problem. It can be useful to evaluate the thermal parameters of the tissue [6]. Therefore, we proposed the simplified approach using 1D thermal model in the Laplace domain. Then we tune the multilayer model to evaluate the thermal parameters of the skin, using the optimization procedures [9]. 


\section{Screening procedure}

The protocol of screening procedure is presented in fig. 1. Cold provocation stimulates the heating process of the skin, especially in its superficial layers. The heating curve was recorded and used to compare it with modelling results. Comparison is performed in Laplace domain. It simplifies the calculation of the model, and makes it fast, allowing the real-time applications [6-8].

The new original element of this research was to use of physical thermal parameters of the multilayer skin structures (thermal conductivities, capacities and perfusions) as the features (indexes, signatures) of the sequence of thermal images for the classification. The approach was successfully tested on psoriasis. The thermographic measurements were performed in clinical conditions in one of the hospital in Lodz. The authors believe that it can be also used for other diseases where the perfusion seems to be an important factor for healthy and unhealthy tissues i.e. melanoma and other skin benign and malignant tumors and cancers.

\section{Active thermography imaging proposed quantitative approach}

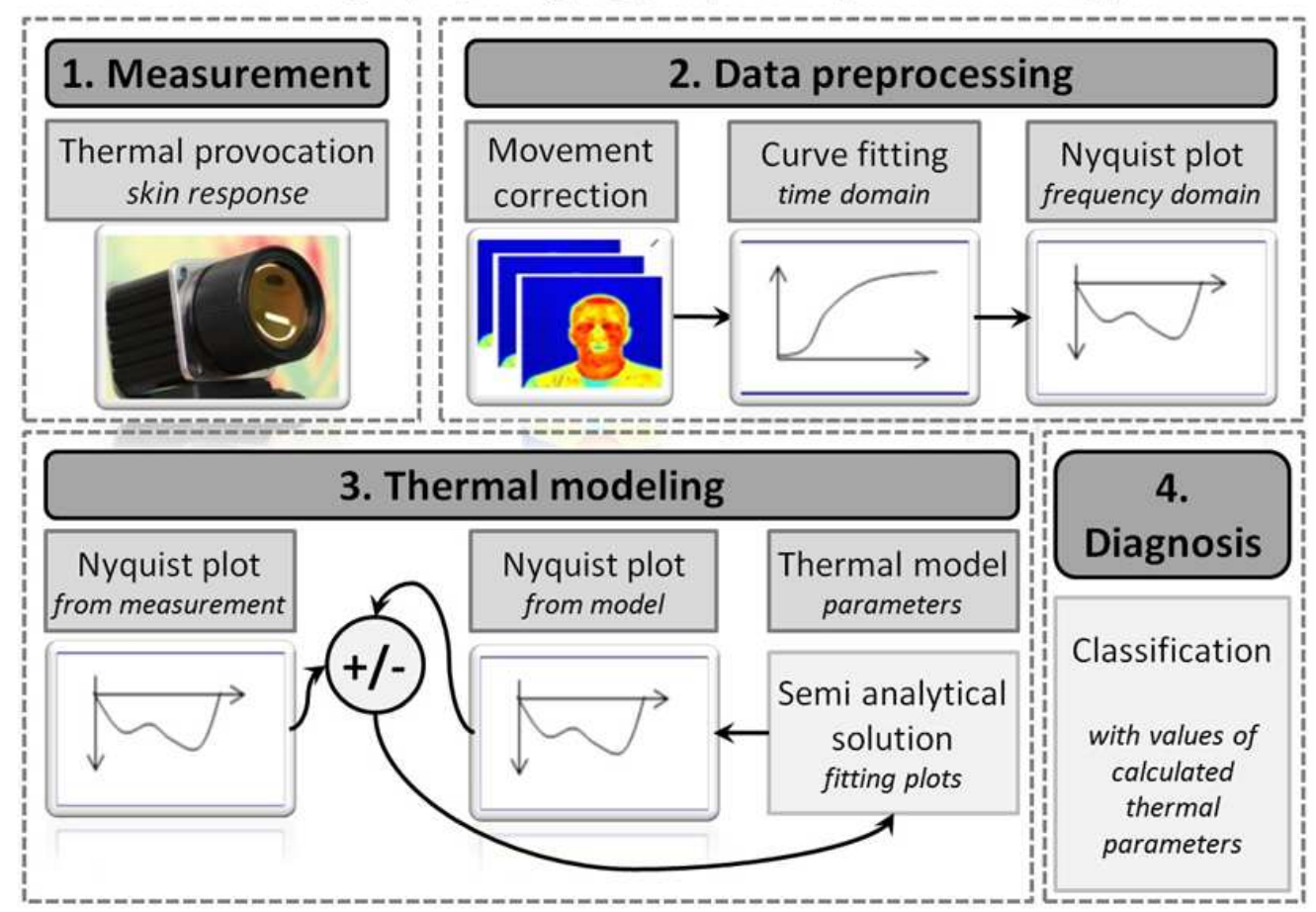

Fig. 1. Overall scheme of the proposed methodology of the screening

\section{Thermal parameters of the skin as the features of the sequences of thermal images}

We modelled the skin as 3-layer structure with perfusion in each of them. We consider epidermis, dermis and hypodermis layers. The thermal parameters of the skin were evaluated using the inverse thermal modeling in Matlab environment - fig. 2. The fitting of the Nyquist plots in Laplace domain was used to get the values of thermal conductivities $-\lambda_{i}$, perfusions $-w_{i}$ and thermal capacities $-C_{t h i}=\rho_{i} c_{p i}$. Developed model of the skin includes also heat transfer coefficient on the upper surface of the skin $-h$, thermal capacity of the blood $-C_{t h b}$, thicknesses $d_{i}$, metabolic heat $-q_{m i}$. Having 3 layers we had 17 thermal parameters. To ensure the integrity of the tissue, we assumed also that the perfusion coefficients have the same value in the second and third layer $-w_{23}$. In the first layer the perfusion is relatively low, we keep the value constant at the level $w_{1}=0.00000001$. This value was found as a value which gives the good fitting of the simulated and measured Nyquist plots. 


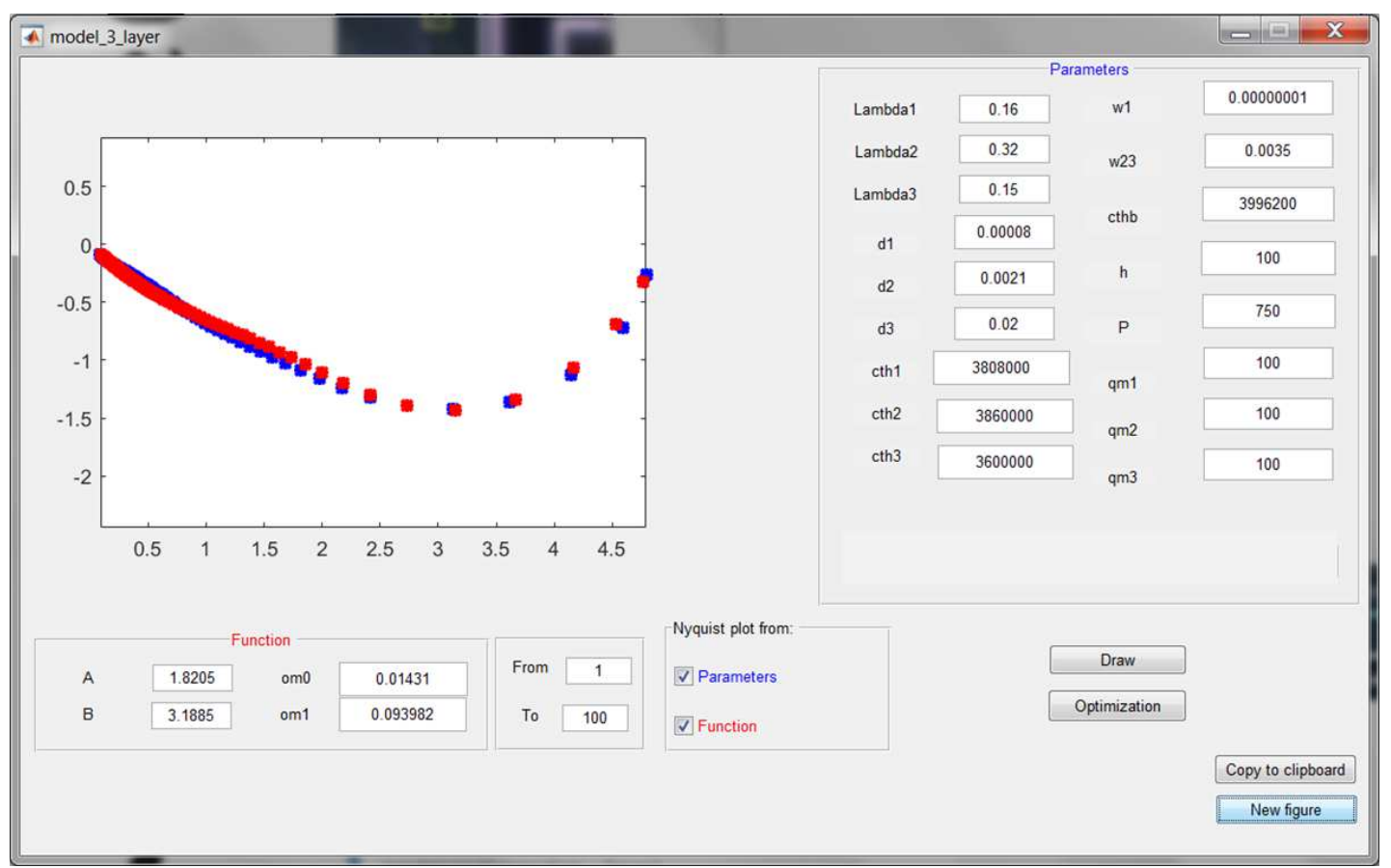

Fig. 2. The main window of the program presenting the fitting of the Nyquist plots obtained by the measurement and simulation with the thermal parameters of the skin

\section{Experimental results}

During the experimental approval of the presented method 9 patients were involved with the recognized psoriasis. We tested our screening approach using skin with the visible inflammation - fig. 3 . The healthy skin of the same patient was used as the reference (control group). Skin was cooled down for few degrees and then the registration of the sequence of thermal images took place till the skin temperature back almost to the initial one before the stress. The algorithm of movement correction had to be applied and then the fitting the measurement curve of temperature rise and the combination of exponential and error function took place in time domain [6-8].

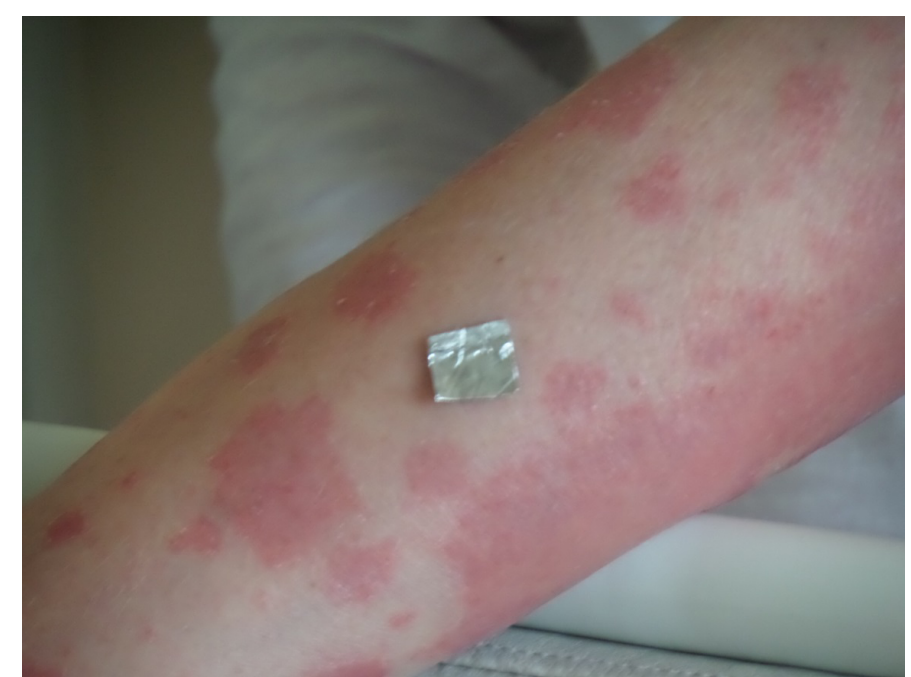

Fig. 3.The arm of a patient with psoriasis and the object for movement correction

After conversion to frequency domain the optimization procedure for fitting the Nyquist plots (simulated and measured - fig. 2) was applied and the values of physical parameters of the skin were calculated. The results are in table 1. The calculated thermal parameters of the tissue should be selected. The first selection can be performed just be comparing the value of each parameter for healthy and unhealthy region of the skin. In fig. 4, one can clearly see the strong correlation between the perfusion in the tissue state of the skin. The skin with high inflammation has significantly higher perfusion. In some case the is 3-5 timer than for skin which looks normal. 
Table 1. The thermal parameters of the 3-layer skin tissue and the perfusion obtained by the fitting of Nyquist plots

\begin{tabular}{|c|c|c|c|c|c|c|c|c|c|c|c|c|c|}
\hline \multicolumn{2}{|c|}{$\lambda_{1}$} & \multicolumn{2}{|c|}{$\lambda_{2}$} & \multicolumn{2}{|c|}{$\lambda_{3}$} & \multicolumn{2}{|c|}{$w_{23}$} & \multicolumn{2}{|c|}{$C_{t h 1}$} & \multicolumn{2}{|c|}{$C_{t h 2}$} & \multicolumn{2}{|c|}{$C_{t h 3}$} \\
\hline H-thy & U-thy & $\mathrm{H}$-thy & U-thy & H-thy & U-thy & $\mathrm{H}$-thy & U-thy & $\mathrm{H}$-thy & U-thy & $\mathrm{H}$-thy & U-thy & $\mathrm{H}$-thy & U-thy \\
\hline 0.14 & 0.34 & 0.25 & 0.25 & 0.59 & 0.29 & 0.00625 & 0.00225 & 2008000 & 3008000 & 2060000 & 3560000 & 3000000 & 2500000 \\
\hline 0.24 & 0.26 & 0.35 & 0.31 & 0.49 & 0.38 & 0.00225 & 0.00625 & 3508000 & 2610000 & 3160000 & 2560000 & 2500000 & 2400000 \\
\hline 0.14 & 0.15 & 0.25 & 0.21 & 0.3 & 0.21 & 0.00125 & 0.00525 & 2610000 & 3010000 & 2560000 & 2760000 & 2900000 & 2900000 \\
\hline 0.14 & 0.2 & 0.25 & 0.25 & 0.1 & 0.17 & 0.00225 & 0.00425 & 3008000 & 2508000 & 3000000 & 2500000 & 2500000 & 2500000 \\
\hline 0.39 & 0.45 & 0.15 & 0.12 & 0.76 & 0.44 & 0.00178 & 0.003 & 3927696 & 3508000 & 3000000 & 3660000 & 3080000 & 3800000 \\
\hline 0.15 & 0.16 & 0.22 & 0.32 & 0.25 & 0.15 & 0.002 & 0.0035 & 3808000 & 3808000 & 3860000 & 3860000 & 3600000 & 3600000 \\
\hline 0.5 & 0.55 & 0.28 & 0.38 & 0.15 & 0.15 & 0.00253 & 0.00553 & 3008000 & 3508000 & 3960000 & 3960000 & 3400000 & 3400000 \\
\hline 0.36 & 0.66 & 0.1 & 0.1 & 0.57 & 0.89 & 0.00236 & 0.0045 & 3708000 & 3908000 & 3060000 & 2560000 & 3500000 & 3700000 \\
\hline 0.15 & 0.44 & 0.23 & 0.7 & 0.10 & 0.80 & 0.003048 & 0.003548 & 3908000 & 3008000 & 3060000 & 3060000 & 3500000 & 3900000 \\
\hline
\end{tabular}

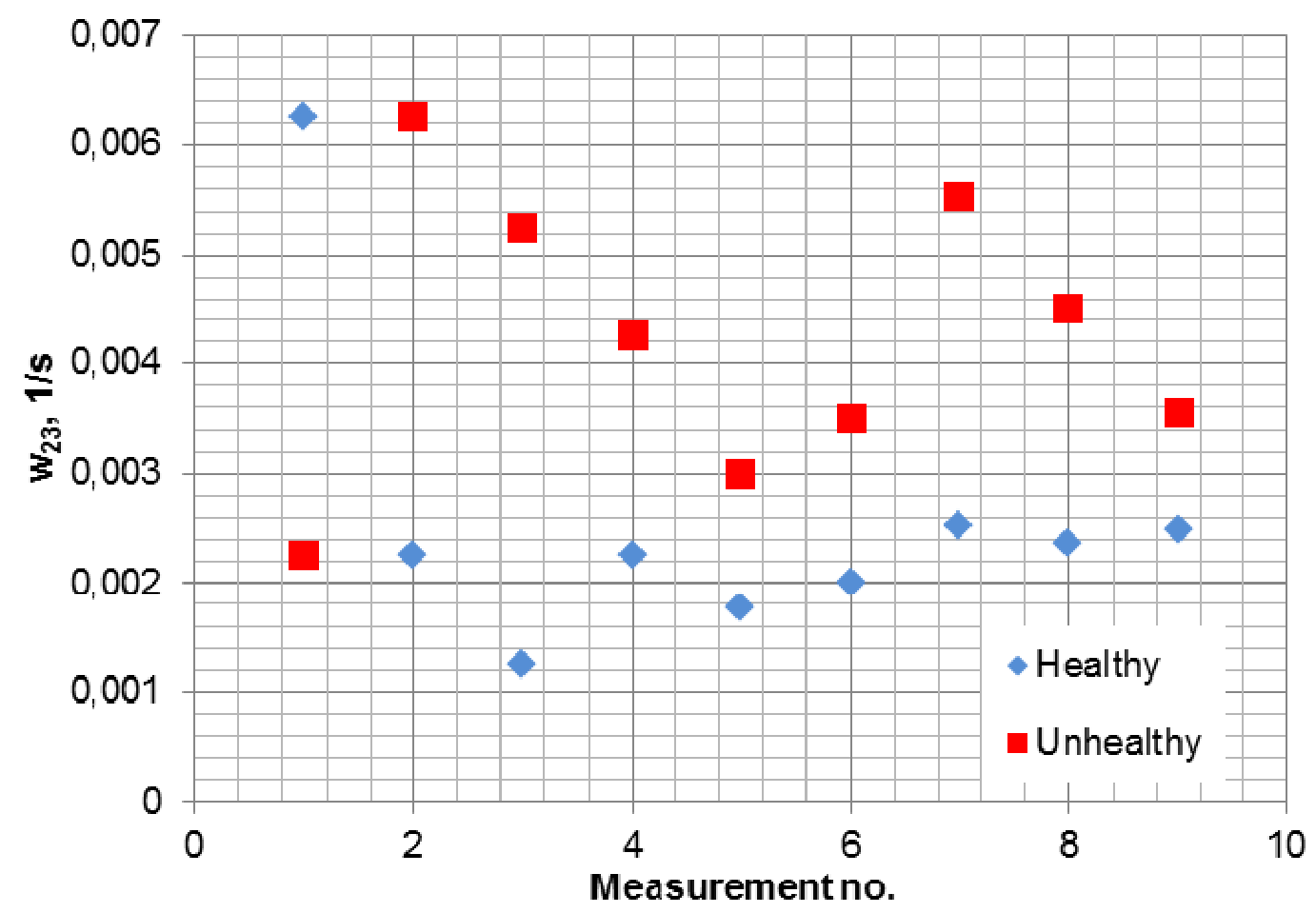

Fig. 4. Perfusion coefficient for healthy and unhealthy skin tissue (psoriasis)

In order to select the other parameters such as thermal conductivities and capacities, one proposed the present them in the relative scale $\underline{p_{\text {healhty }}-p_{\text {unhealhty }}}$. Then, one uses the threshold separating the positive and negative values. $p_{\text {healhty }}$

The obtained results for thermal conductivities and capacities are not so clear as for blood perfusion. However, one can notice in fig. 5 for most of the cases the $1^{\text {st }}$ layer (epidermis) has a lower thermal conductivity while the $3^{\text {rd }}$ one (hypodermis) conducts the better the heat when the skin is in healthy state. The cold bodies typically are more resistive to the heat, and epidermis is cooled the most. The thermal capacities are randomly distributed. This fact can be explained by statement that the tissue consists of the water in majority and has one of the highest thermal capacity comparing to other materials. 


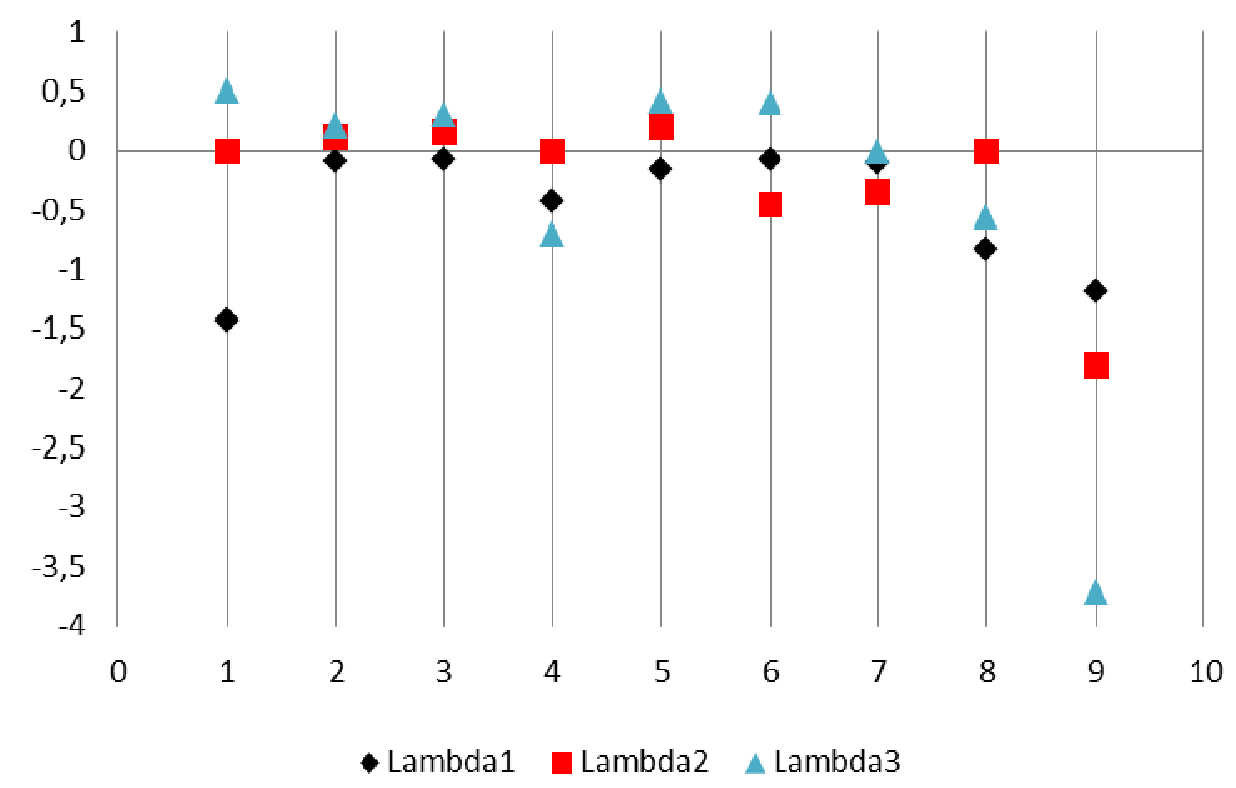

Fig. 5. Thermal conductivity for epidermis $\left(\lambda_{1}\right)$, dermis $\left(\lambda_{2}\right)$ and hypodermis $\left(\lambda_{3}\right)$ layers for healthy and unhealthy skin tissue (psoriasis) in the relative scale

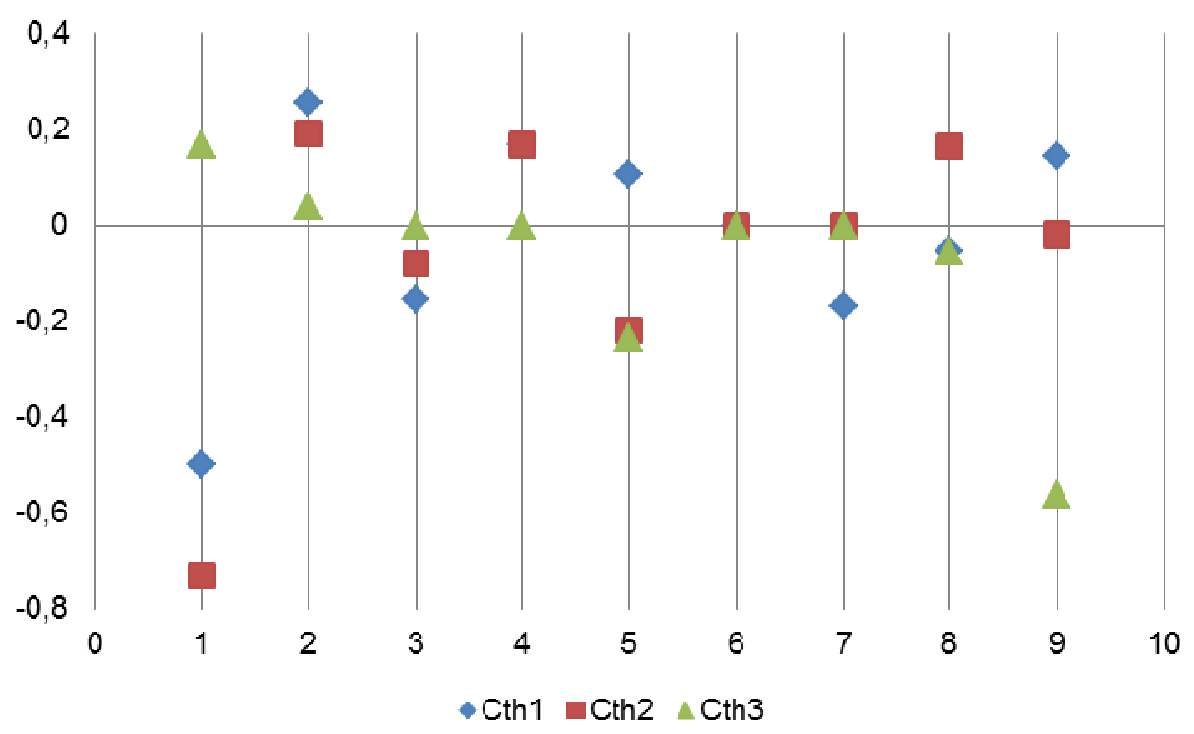

Fig. 6. Thermal capacities for epidermis $\left(C_{t h 1}\right)$, dermis $\left(C_{t h 2}\right)$ and hypodermis $\left(C_{t h 3}\right)$ layers for healthy and unhealthy skin tissue (psoriasis) in the relative scale

Finally, we decided to calculate the thermal time constants or/and angular frequencies corresponding to each layer. The thermal angular frequency depends on the thickness of the layer and its thermal parameters as presented by equation (4).

$$
\omega_{i}=\frac{\lambda_{i}}{d_{i}^{2} \rho_{i} c_{p i}}
$$

The calculated values of angular frequencies are presented in table 2 . As one can see in table 2 , the superficial layers reacts faster to the heat. It is the reasonable result because of the lower thickness of the superficial skin layers. Another conclusion drawn by analyzing the data in table 2, is that in the most of cases the tissue with the inflammation reacts faster than healthy one. In our opinion, this is because of the higher perfusion. 
Table 2. Angular frequencies for each layer of 3-layer structure of the screen.

\begin{tabular}{|r|r|r|r|r|r|}
\hline \multicolumn{2}{|c|}{$\mathbf{1}$ (epidermis) } & \multicolumn{2}{c|}{$\boldsymbol{\omega 2}$ (dermis) } & \multicolumn{2}{c|}{$\boldsymbol{\omega}$ (hypodermis) } \\
\hline Healthy & Unhealthy & Healthy & Unhealthy & Healthy & \multicolumn{1}{c|}{ Unhealthy } \\
\hline 10.89392 & 17.66124 & 0.007585 & 0.011236 & 0.007867 & 0.001813 \\
\hline 10.68985 & 15.56513 & 0.110759 & 0.121094 & 0.00049 & 0.000396 \\
\hline 8.381226 & 7.786545 & 0.024414 & 0.019022 & 0.000259 & 0.000181 \\
\hline 7.272274 & 12.46013 & 0.018896 & 0.022676 & 0.0004 & 0.00068 \\
\hline 15.47027 & 20.04347 & 0.063591 & 0.177322 & 0.000621 & 0.000289 \\
\hline 6.154806 & 6.565126 & 0.014249 & 0.020725 & 0.000174 & 0.000104 \\
\hline 25.97241 & 24.49758 & 0.017677 & 0.118469 & 0.00011 & 0.00011 \\
\hline 15.1699 & 26.38818 & 0.090777 & 0.120229 & 0.000724 & 0.000267 \\
\hline 8.90821 & 22.64794 & 0.068871 & 0.228758 & 0.00017 & 0.000513 \\
\hline
\end{tabular}

Reassuming, we propose to use the following thermal parameters of the 3-layer skin tissue for the classification to distinguish the pathological and physiological state:

- combined perfusion in dermis and hypodermis,

- thermal conductivities,

- angular frequencies/thermal time constants.

\section{Conclusions}

This paper presented the protocol of selecting the physical thermal parameters of the 3-layer skin tissue for the classification the pathologies. We think that perfusion in dermis and hypodermis, chosen thermal conductivities and time constants would be the good candidates for the classification. The classification using various methods will be made and the results will be presented soon. We believe that the proposed method of screening will became an objective and helpful tool in the diagnosis of various skin pathologies.

\section{Acknowledgments}

This work was supported by the National Science Centre Poland, project no. UMO-2013/11/N/ST7/02630.

\section{REFERENCES}

[1] Pennes $\mathrm{HH}$. Analysis of tissue and arterial blood temperatures in the resting human forearm. Journal of Applied Physiology 1948; 1(2).

[2] Zolfaghari A, Maerefat M. Bioheat transfer, developments in heat transfer, Dr. Marco Aurelio Dos Santos Bernardes (Ed.), 2011, ISBN: 978-953-307-569-3, InTech, Available from: http://www.intechopen.com/books/developments-in-heat-transfer/bioheat-transfer.

[3] Jasiński M. Modelling of 1D bioheat transfer with perfusion coefficient dependent on tissue necrosis. Scientific Research of the Institute of Mathematics and Computer Science, Czestochowa University of Technology, Vol. 7, 2008.

[4] Khanafer K, Vafai K.Synthesis of mathematical models representing bioheat transport. Advances in Numerical Heat Transfer, Vol III, Chap. 1, pp. 1- 28, CRC Press, New York, 2009.

[5] $\mathrm{Ng}$ EYK, Tan H, Ooi E, Boundary element method with bioheat equation for skin burn injury, Burns 35:987-997, 2009.

[6] Strakowska M, De Mey G, Wiecek B, Strzelecki M. A three layer model for the thermal impedance of the human skin: modelling and experimental measurements. J. Mech. Med. Biol. 2015;15.

[7] Maria Strąkowska, Michał Strzelecki, Andrzej Kaszuba, Novel methodology of medical screening using IR thermography, SPA 2014. Signal Processing. Algorithms, Architecture, Arrangements and Applications. Conference proceedings, s.172-175,2014.

[8] Maria Strakowska, Andrzej Kaszuba, Boguslaw Wiecek, Michal Strzelecki and Gilbert De Mey, System and software for thermal images screening in medicine - application to psoriasis, Quantitative InfraRed Thermography, 2014.

[9] M. R. K. Mookiaha, U. Rajendra Acharyaab \&E.Y.K. Ng, Data mining technique for breast cancer detection in thermograms using hybrid feature extraction strategy, Quantitative InfraRed Thermography Journal, Volume 9, Issue 2, 2012. 